\title{
Tecnura
}

INVESTIGACIÓN

\section{Efecto del gas en la velocidad de onda de corte de suelos arenosos densificados con explosivos}

\section{Effect of gas on shear wave velocity of sandy soils densified with explosives}

\author{
Carlos Alberto Vega-Posada, Alfonso Mariano Ramos-Cañón², Edwin Fabián García Aristizábal ${ }^{3}$
}

Fecha de recepción: 10 de marzo de 2016

Fecha de aceptación: 23 de noviembre de 2016

Cómo citar: Vega-Posada, C.A.; Ramos-Cañón, A.M. y García-Aristizábal, E.F. (2017). Efecto del gas en la velocidad de onda de corte de suelos arenosos densificados con explosivos. Revista Tecnura, 21(51), 67-80. doi: 10.14483/udistrital.jour.tecnura.2017.1.a05

\section{Resumen}

Contexto: Los ensayos de velocidad de onda de corte $(\mathrm{Vs})$, son comúnmente utilizados para estimar el incremento en resistencia de suelos densificados con explosivos. En algunos casos históricos los ensayos de $\vee s$ realizados después del proceso de mejoramiento de suelo no muestran un incremento significativo en la resistencia del suelo, aun cuando la superficie del terreno se asienta más de 0,50 m. Se cree que esta respuesta se debe a la presencia de gas en la masa de suelo.

Método: En este trabajo se presentan los resultados de ensayos triaxiales monotónicos realizados en muestras de suelos arenosos gaseosos medianamente densos, para evaluar el efecto del gas ocluido en la respuesta a la velocidad de onda de corte en arenas densificadas con explosivos. Muestras de arena fueron recolectadas de un depósito de arena suelta localizado in Carolina del Sur, Estados Unidos, y el cual fue densificado in situ con explosivos. Las muestras fueron consolidadas a las condiciones de esfuerzo efectivo in situ, las cuales se consideran representativas de las condiciones de esfuerzo en el momento de realizar la densificación con explosivos.
Resultados: Los resultados de los ensayos triaxiales, realizados bajo condiciones no-drenadas globales, mostraron que, independientemente de la densidad de la muestra, el gas hace que los valores de velocidad de onda de corte obtenidos para las arenas gaseosas se aproximen a los valores de velocidad de onda de corte obtenidos en las muestras saturadas ensayadas bajo condiciones drenadas. Además, este comportamiento tiende a ser más pronunciado a medida que el suelo es más denso.

Conclusiones: Esta respuesta podría ofrecer algunas luces sobre porqué la velocidad de corte no se incrementa significantemente en suelos densificados con explosivos aun cuando la densidad se incrementa considerablemente.

Palabras clave: arenas sueltas, densificación con explosivos, licuación, mejoramiento de suelos, velocidad de onda de corte

\begin{abstract}
Context: Shear wave velocity tests (Vs) are commonly used to estimate the increase in resistance of explosive densified soils. In some historical cases, Vs tests performed after the soil improvement process
\end{abstract}

1 Ingeniero civil, doctor en Ingeniería Civil. Profesor asistente, Grupo de Investigación en Infraestructura (GII), Escuela Ambiental, Universidad de Antioquia, Calle 67 \# 53-108. A. A. 1226, Medellín, Colombia. Contacto: carlosa.vega@udea.edu.co

2 Ingeniero civil, doctor en Ingeniería. Profesor asociado, Instituto Geofísico, Facultad de Ingeniería, Pontificia Universidad Javeriana, Calle 67 \# 53-108. A. A. 1226, Bogotá, Colombia. Contacto: a-ramos@javeriana.edu.co

3 Ingeniero civil, doctor en Ingeniería Civil. Profesor Asociado, grupo de Investigación en Infraestructura (GII), Escuela Ambiental, Universidad de Antioquia, Calle 67 \# 53-108. A. A. 1226, Medellín, Colombia. Contacto: edwin.garcia@udea.edu.co 
do not show a significant increase in soil resistance, even though the soil surface sits more than $0.50 \mathrm{~m}$. It is believed that this response is due to the presence of gas on the soil mass.

Method: This paper presents the results of monotonic triaxial tests performed on samples of dense gaseous sandy soils to evaluate the effect of occluded gas on the response to the shear wave velocity in densified sands with explosives. For sand sampling, it was collected from a loose sand deposit located in South Carolina, USA. These samples were densified in-situ with explosives, and consolidated to the insitu effective stress conditions, which are considered representative in the conditions of effort at the moment of the densification with explosives.
Results: Triaxial tests were performed under global non-drained conditions. The results of these tests show that gas causes the shear wave velocity values obtained for the gaseous sands to approximate the shear wave velocity values obtained in the saturated samples tested under drained conditions. In addition, behavior tends to be more pronounced as the soil is denser.

Conclusions: These response may offer some insights as to why the shear wave velocity does not increase significantly in densified soils with explosives, even though the density increases considerably.

Keywords: Loose sands, blast densification, liquefaction, soil improvement, shear wave velocity

\section{INTRODUCCIÓN}

La densificación con explosivos es una técnica común usada para densificar grandes áreas que poseen horizontes de suelos potencialmente licuables, con el objeto de prevenir o mitigar el efecto de licuación y flujo inducido por fuerzas sísmicas. La principal limitación de esta técnica es que el diseño es principalmente empírico y no existe un procedimiento teórico bien establecido y disponible para conducir el diseño de este tipo de densificación, y para determinar el número de series de explosiones necesarias para alcanzar la densidad objetivo (Gohl, Jefferies, Howie y Diggle, 2000; Ramos, 2015). La densificación con explosivos consiste en colocar cargas explosivas dentro de un horizonte de suelo que necesita ser densificado y detonarlas en múltiples intervalos para generar una carga cíclica. La onda de alta presión rompe cualquier vínculo entre las partículas ocasionado por procesos de cementación y adicionalmente genera exceso de presión de poros. A medida que el exceso de presión de poros se incrementa, el esfuerzo efectivo de confinamiento disminuye hasta cero y se logra la licuación del material. Después de darse la licuación y a medida que la presión de poros se disipa, el suelo alcanza un estado más denso y el esfuerzo efectivo de confinamiento regresa a los valores previos al proceso de densificación. Dependiendo de la distribución granulométrica, la densidad inicial de la arena y del programa de densificación con explosivos, se pueden lograr deformaciones axiales verticales hasta de $4 \%$ en una serie de detonación (Vega-Posada, 2012). Es posible alcanzar mayores niveles de compactación implementando series adicionales de detonaciones (Narsilio, 2006; Narsilio, Santamarina, Hebeler y Bachus, 2009; Vega-Posada, 2012; Vega-Posada, Zapata-Medina y García-Aristizábal, 2014).

Las principales limitaciones de la técnica de densificación con explosivos consisten en que esta metodología de diseño es ampliamente empírica y la verificación de los niveles de densificación muestra resultados contradictorios en algunos casos de estudio. En estos últimos, inclusive, aunque el horizonte de suelo ha sido densificado y la superficie del suelo se asienta casi inmediatamente después de la explosión, las verificaciones realizadas con equipos de campo, como: ensayo de penetración de cono (cone penetration test, CPT), ensayo de penetración estándar (standard penetration test, SPT) y el ensayo de velocidad de onda 
cortante (shear wave velocity test, $\mathrm{V}_{\mathrm{s}}$ ), sugieren que la masa de suelo no se ha mejorado. Estos resultados traen como consecuencia algunas inquietudes acerca del comportamiento posterior del suelo y genera dudas sobre si la arena suelta ha sido mejorada hasta el punto de evitarse la licuación o no.

Debido a que se producen y liberan grandes cantidades de gas durante el proceso de densificación, y una parte de este gas permanece atrapado en el suelo por muchos años (Finno, Gallant y Sabatini, 2016; Okamura, Ishihara y Tamura, 2006; Okamura et al., 2011; Vega-Posada, 2012; Yegian, Eseller-Bayat, Alshawabkeh y Ali, 2007), los depósitos de arena densificados con explosivos pueden ser clasificados como suelos gaseosos (Ramos, Felipe y Vega-Posada, 2016; Vega-Posada, Finno y Zapata-Medina, 2014). Los suelos gaseosos son un tipo especial de suelo donde el gas (i.e., aire) existe en una condición ocluida y esta fase gaseosa no está conectada con la atmósfera (Nageswaran, 1983). En este artículo se presentan los resultados experimentales de un programa de laboratorio conducido con el objeto de evaluar el efecto del gas ocluido en la velocidad de onda de corte en arenas densificadas con explosivos. Para este propósito, se realizaron un total de 20 ensayos triaxiales monotónicos en muestras de arena alteradas medianamente densas. Los resultados mostraron que, inclusive, una pequeña cantidad de gas modifica la respuesta de la muestra. Se encontró que debido a la presencia de gas, la velocidad de onda de corte Vs no se incrementa considerablemente a medida que la densidad de la muestra se incrementa, lo que podría explicar resultados obtenidos en depósitos de arena mejoradas con explosivos.

\section{METODOLOGIA}

Se preparó un programa experimental de laboratorio, consistente en una serie de ensayos triaxiales, con el propósito de evaluar el efecto de las burbujas de gas ocluidas en el comportamiento mecánico y en la velocidad de onda de corte de arenas densificadas con explosivos. Las muestras de arena fueron recolectadas de un depósito donde se construye un relleno sanitario, y fueron consolidadas anisotrópicamente a niveles de esfuerzos in situ y relaciones de vacío representativas del horizonte de suelo en estudio. Para cada relación de vacíos, se prepararon muestras saturadas y gaseosas con grados de saturación entre 83,5 y 98 \% para cuantificar el efecto del gas en la respuesta de $V s$ posterior a la densificación. Debido a que el propósito del programa experimental es evaluar la respuesta de Vs en arenas a medida que estas se densifican por medio del uso de los explosivos, se consideraron solo muestras con relaciones de vacío por debajo de la línea de estado crítico (más denso que la densidad crítica). Tanto el montaje experimental como la técnica utilizada para la preparación de las muestras gaseosas (i.e., sedimentación en agua) limitaron el programa experimental de las muestras de arena medianamente densas debido a que no era posible obtener muestras de mayores relaciones de vacíos con esta técnica. Adicionalmente, el programa experimental incluyó una serie de ensayos triaxiales a compresión en muestras menos densas que la densidad crítica de la arena para determinar la línea de estado crítico (LEC). Esta última se usó para evaluar la respuesta de los ensayos triaxiales monotónicos desde el punto de vista conceptual del estado crítico de suelos.

Aunque el nitrógeno es el gas atrapado que predomina y permanece por más tiempo en el suelo después del proceso densificación con explosivos, este no es fácil de disolver en agua y requiere de altas presiones para poder alcanzar un grado deseado de saturación, esto hace que el nitrógeno sea impráctico para este tipo de trabajos de laboratorio. Por tanto, en lugar de nitrógeno, se utilizó agua carbonatada para preparar las muestras de arena gaseosa. El agua carbonatada (agua saturada con dióxido de carbono) es fácilmente obtenida en el laboratorio, no es corrosiva, no es inflamable y requiere de presiones muy bajas para alcanzar un grado de saturación deseado en las muestras de suelo. En éste tipo de muestras, la resistencia al cortante no drenada en condiciones saturadas es un poco 
menor (i.e., un poco más conservativa) que las resistencias obtenidas con muestras saturadas con nitrógeno (Grozic, Imam, Robertson y Morgenstern, 2005). Sin embargo, la tendencia general de los resultados con muestras que contienen gas es la misma, independiente de que el proceso de saturación se haga con dióxido de carbono o nitrógeno.

\section{Descripción del material}

Las muestras de arena se recolectaron de un horizonte de suelo sobre el cual existe un relleno sanitario. La zona de estudio está localizada cerca de la ciudad de Charleston (Corolina del Sur, Estados Unidos). El horizonte de suelo se encuentra a unas profundidades entre 7,5 y 13 m y en él se realizó un proceso de densificación con explosivos con el objeto de incrementar la resistencia del suelo a la licuación y flujo (véase Vega-Posada et al., 2014). Las muestras fueron tomadas a una profundidad aproximada de 10 metros. La figura 1 presenta la curva granulométrica de la arena. El porcentaje de finos que pasa la malla No. 200 para granulometría seca y húmeda fue de 1,5 y $7 \%$, respectivamente. El coeficiente de uniformidad $\left(C_{u}\right)$ y de curvatura $\left(C_{c}\right)$ fueron 1,72 y 1,06 , respectivamente. Las relaciones de vacíos mínima $\left(e_{\min }\right)$ y máxima $\left(e_{\max }\right)$ fueron 0,62 y 1,05 , respectivamente; la gravedad específica $\left(G_{s}\right)$ fue de 2,66. De acuerdo con el sistema unificado de clasificación de suelos (SUCS), el material se clasifica como una arena pobremente gradada (SP).

\section{Equipo para ensayos}

La figura 2 muestra un esquema del aparato triaxial utilizado para la realización de los ensayos. Estos se ejecutaron en un sistema de ensayo triaxial dinámico avanzado (Advanced Dynamic Triaxial Testing System, DYNTTS) desarrollado por GDS Intruments. Este sistema combina una celda triaxial de 2 MPa con un pistón dinámico capaz de aplicar fuerzas axiales hasta de $10 \mathrm{kN}$ o deformaciones axiales hasta de $100 \mathrm{~mm}$ con frecuencias hasta de $2 \mathrm{~Hz}$. El sistema consiste de un controlador de presión de poros o de volumen y un controlador de la presión de cámara o de volumen con rangos de presión entre 0 y $200 \mathrm{kPa}$ y capacidad volumétrica de $200 \mathrm{~cm}^{3}$. Los controladores pueden aplicar cambios de presiones y volúmenes de $0,2 \mathrm{kPa}$ y $1 \mathrm{~mm}^{3}$, respectivamente. La presión de poros se mide por medio de un transductor de presión de poros localizado en la base de la muestra.

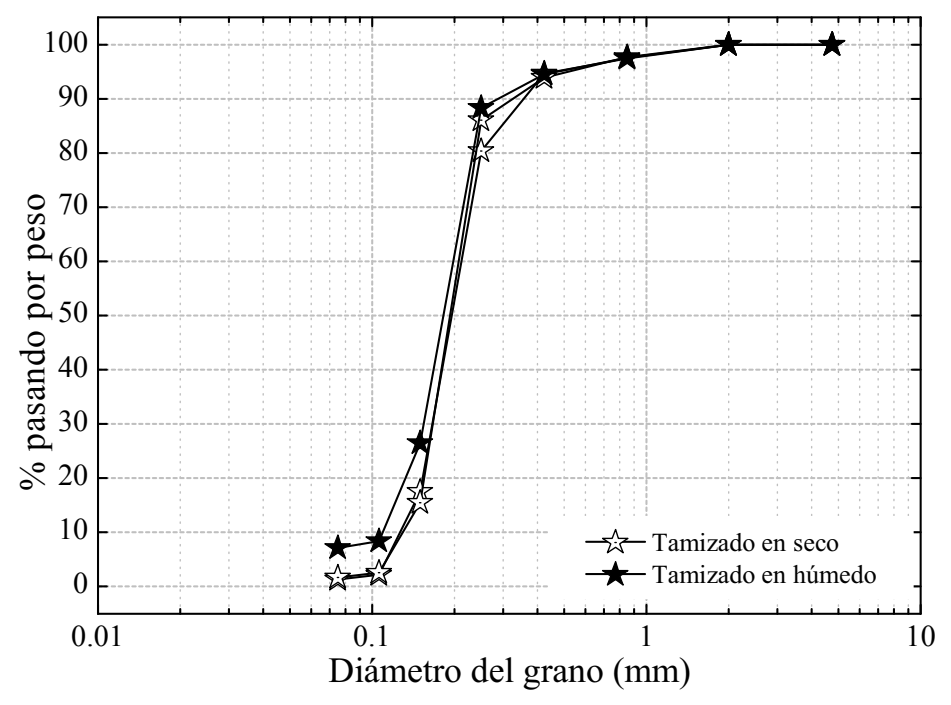

Figura 1. Curva de distribución granulométrica típica de la arena

Fuente: elaboración propia. 


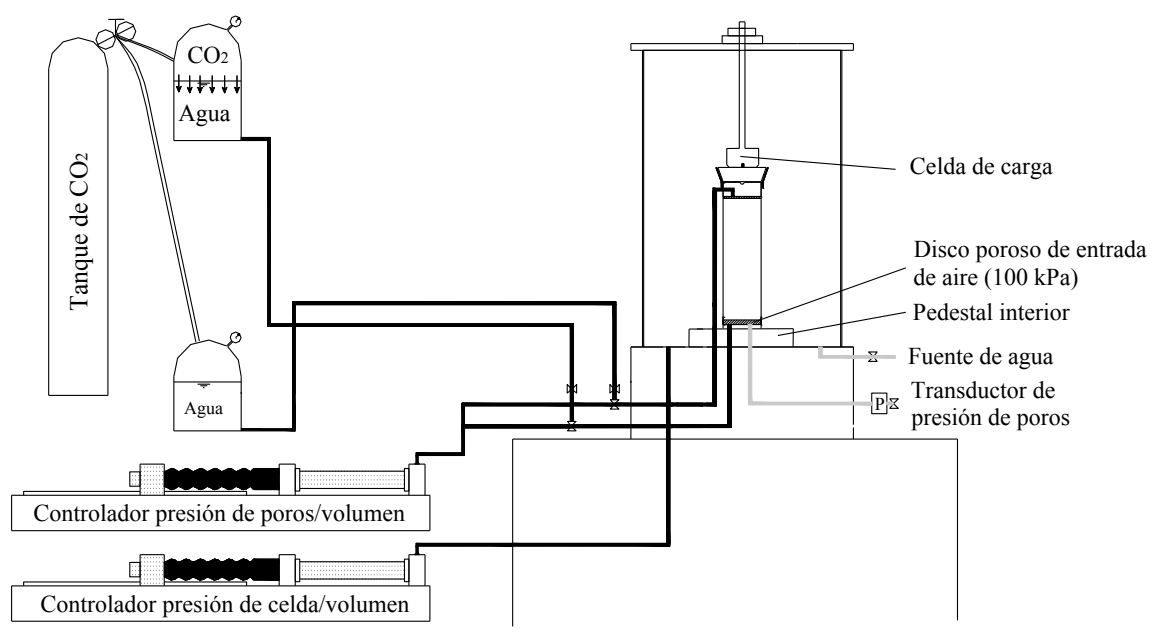

Figura 2. Diagrama esquemático del aparato triaxial

Fuente: elaboración propia.

\section{Preparación de la muestra}

En el programa de ensayos planeado, las muestras de arena gaseosas y las muestras de arena saturadas de control usadas para los ensayos triaxiales monotónicos se prepararon usando la técnica de sedimentación en agua. Se considera que esta técnica de preparación del suelo da como resultado muestras uniformes que reproducen acertadamente la respuesta de muestras inalteradas tomadas de depósitos de suelos marinos (Ghionna y Porcino, 2006; Vaid y Sivathayalan, 2000; Vaid, Sivathayalan y Stedman, 1999). Las muestras de suelo utilizadas para determinar la línea de estado crítico fueron preparadas mediante la técnica de compactación húmeda, debido a que no es posible obtener muestras con relaciones de vacíos mayores por medio de la técnica de sedimentación en agua; además, es necesario tener muestras de arenas sueltas con el propósito de obtener una línea de estado crítico confiable. Para la preparación de las muestras se siguieron los procedimientos propuestos por Chaney y Mulilis (1978) y Ladd (1978); adicionalmente, se tiene en cuenta que, aunque la ruta de esfuerzos efectiva y la respuesta esfuerzo-deformación es altamente influenciada por la preparación de la muestra (Vaid y Sivathayalan,
2000; Vaid et al., 1999), la respuesta en un estado permanente a altos niveles de esfuerzo no se ve influenciada por este proceso. Las muestras preparadas bajo diferentes técnicas convergen en la misma curva del espacio e- $q-p^{\prime}$, mientras la respuesta del suelo sea contractiva (Been, Jefferies y Hachey, 1991; Castro, Seed, Keller y Seed, 1992; Poulos, Castro y France, 1985; Verdugo e Ishihara, 1996). Been, Jefferies y Hachey (1991) concluyeron que las muestras de suelo preparadas por la metodología sedimentación en agua y compactación húmeda convergen en la misma línea de estado crítico.

Las dimensiones de las muestras utilizadas fueron aproximadamente de $50 \mathrm{~mm}$ de diámetro y $100 \mathrm{~mm}$ de altura. El procedimiento para la preparación de las muestras fue similar al presentado previamente por Chern $(1981 ; 1985)$. En la preparación de la muestra, un molde compuesto por tres partes y una membrana se colocaron en el pedestal inferior del triaxial. Posteriormente, se generó un vacío de $20 \mathrm{kPa}$ en el molde para hacer que la membrana se adhiriera internamente a este durante la preparación de la muestra. El molde se Ilenó con agua de-aireada y luego se depositó la arena bajo condiciones saturadas.

La arena fue depositada en la parte baja del molde (cerámica porosa) por medio de un embudo, 
el cual se levantaba lentamente a medida que se llenaba el molde para permitir la sedimentación de las partículas de arena. Durante este procedimiento se mantuvo la boquilla del embudo aproximadamente $10 \mathrm{~mm}$ por encima de las partículas previamente sedimentadas. Después de alcanzar la altura objetivo de la muestra, se instaló el tope superior dejando la línea de drenaje abierta, de tal manera que se permitiera la salida del aire atrapado y se evitaran excesos de presión de poros sobre la muestra por la instalación de este elemento. Para la preparación de las muestras más densas se siguió el mismo procedimiento pero aplicando una pequeña vibración al molde. La ventaja que ofrece esta técnica de preparación es que se puede realizar el proceso en condiciones saturadas (sin contacto con el aire), resultando generalmente en muestras con valores B mayores que 0,96. Después de terminada la preparación de la muestra, se aplica un vacío de $20 \mathrm{kPa}$ a través de las caras superior e inferior para remover el molde y colocar la cámara triaxial para ser Ilenada con agua de-saturada. Una vez se coloca la cámara triaxial, se aplica una presión de confinamiento de $20 \mathrm{kPa}$ para remover el vacío aplicado a la muestra.

Para la preparación de las muestras de arenas gaseosas, la presión de poros se incrementó gradualmente hasta valores entre 300 y $450 \mathrm{kPa}$, mientras se mantenía el esfuerzo efectivo de confinamiento $\left(\sigma_{c}^{\prime}=20 \mathrm{kPa}\right)$. Una vez se alcanzaba un valor de $B$ mayor a 0,96 , se consolidaba la muestra a los niveles de esfuerzo efectivo in situ y se reemplazaba el fluido con agua saturada con dióxido de carbono. El reemplazo del fluido se hizo por medio de un sistema de circulación similar al presentado por Amaratunga y Grozic (2009). La figura 2 presenta un esquema del sistema de circulación el cual consiste en dos contenedores Ilenados parcialmente con agua de-aireada; el primer contenedor está colocado a una altura de $0,8 \mathrm{~m}$ por encima de la cámara triaxial, el segundo contenedor está colocado a una altura de 0,8 $\mathrm{m}$ por debajo de la cámara triaxial, generando así una diferencia en la cabeza de altura de 1,6 m. Los contenedores superior e inferior fueron conectados a las líneas de drenaje superior e inferior, respectivamente, para crear un flujo ascendente del agua durante el reemplazo del fluido. Para producir el agua saturada con dióxido de carbono, los contenedores se presurizaron con un tanque de dióxido de carbono y la presión se mantuvo por 24 horas, tiempo que se considera suficiente para saturar completamente el agua con dióxido de carbono (Knai, 2011). Adicionalmente, los contenedores fueron presurizados a la misma presión de poros aplicada a la muestra para mantener el esfuerzo efectivo constante durante el reemplazo del fluido. Para asegurar un buen reemplazo del fluido por el agua saturada con dióxido de carbono, se pasó un volumen de este fluido a través de la muestra aproximadamente igual a tres veces el volumen de los poros. Este proceso tomó de 3 a 4 días y fue realizado bajo un esfuerzo efectivo principal normal de $100 \mathrm{kPa}$. Una vez se reemplazó el fluido la presión de poros se redujo gradualmente hasta igualar las presiones de poro in situ, mientras se aplicaba un esfuerzo efectivo constante y haciendo que el dióxido de carbono saliera de la solución en forma de burbujas ocluidas, reduciendo así el grado de saturación de la muestra. Para alcanzar diferentes grados de saturación se ajustó la presión a aquella a la cual el dióxido de carbono se disuelve en agua.

Aunque el pedestal inferior del aparato triaxial contiene una cerámica porosa con un valor de entrada de aire de 100 kPa para prevenir que el dióxido de carbono salga de la solución, la línea de drenaje inferior fue lavada con agua de-aireada para remover cualquier contenido de agua saturada con dióxido de carbono o burbujas de gas que existiesen bajo la cerámica porosa. Este procedimiento se realizó previo a la desaturación y antes de las cargas cíclicas para asegurar que las lecturas de la presión de poros en los pasos siguientes fueran precisas y confiables.

Finalmente, las muestras fueron ensayadas en condiciones no drenadas. La velocidad de corte, tanto para las muestras saturadas como para las muestras gaseosas, fue de $0,2 \mathrm{~mm} / \mathrm{min}$, lo que corresponde a una deformación unitaria de $0,22 \% / \mathrm{min}$. 


\section{Programa experimental de ensayos triaxiales}

La tabla 1 muestra el listado de los ensayos que forman parte del programa experimental. Se realizaron un total de 20 ensayos triaxiales sobre muestras alteradas de arena de densidad media saturada y gaseosa. De estos ensayos, quince (15) fueron en condiciones no drenadas bajo carga axial monotónica para cuantificar la respuesta del suelo en función de la relación de vacíos (e), y el grado de saturación (S). Los otros cinco (5) ensayos fueron realizados para determinar la posición de la línea de estado crítico.

Las muestras, excepto aquellas utilizadas para la localización de la línea de estado crítico, fueron consolidadas anisotropicamente a esfuerzos efectivos promedio normales de $100 \mathrm{kPa}\left(\sigma_{3 \mathrm{c}}^{\prime}=\right.$ $82 \mathrm{kPa}, \sigma_{1 \mathrm{c}}^{\prime}=136 \mathrm{kPa}$, and $\mathrm{k}_{\mathrm{o}}=0,6$ ); esfuerzos que son representativos de las condiciones de esfuerzos del horizonte de arena suelta a la profundidad de muestreo (aproximadamente $10 \mathrm{~m}$ ). Los ensayos triaxiales se dividieron en tres grupos para cuantificar la respuesta monotónica del suelo en función de la relación de vacíos y el grado de saturación. Tanto las relaciones de vacío usadas como los grados de saturación son representativos de las condiciones de la arena donde se realizó la densificación con explosivos. Las muestras preparadas por la técnica de compactación húmeda se usaron para definir la línea de estado crítico y para comparar los resultados obtenidos con las muestras preparadas con la técnica de sedimentación en agua. En la tabla 1 se presenta el número del ensayo, el tipo de ensayo (saturado o gaseoso), la relación de vacíos después de la consolidación, el esfuerzo efectivo medio normal después de la consolidación, las condiciones de carga (D: drenada o U: no drenada), el grado de saturación después de la carga y el propósito del ensayo.

Tabla 1. Listado de los ensayos que componen el programa de laboratorio

\begin{tabular}{|c|c|c|c|c|c|c|}
\hline Muestra & Tipo de ensayo & $\mathbf{e}_{\text {cons. }}=\mathbf{e}_{\text {sh. }}$ & $\mathrm{p}^{\prime}(\mathrm{kPa})$ & Drenaje & $\mathrm{S}(\%)$ & Propósito \\
\hline MT-01 & Saturado & 0.972 & 84 & CAU & 100 & CSL \\
\hline MT-02 & Saturado & 0.845 & 502 & CAU & 100 & CSL \\
\hline MT-03 & Saturado & 0.785 & 1526 & CAU & 100 & CSL \\
\hline MT-04 & Saturado & 0.993 & 100 & CAU & 100 & CSL \\
\hline MT-05 & Saturado & 0.826 & 697 & CAU & 100 & CSL \\
\hline WP-06 & Saturado & 0.817 & 100 & CAD & 100 & \multirow{4}{*}{$\begin{array}{l}\text { Resistencia } \\
\text { - al cortante } \\
-\mathrm{e}_{\text {aver. }}=0.83\end{array}$} \\
\hline WP-07 & Gaseoso & 0.820 & 100 & CAU & 94 & \\
\hline WP-08 & Gaseoso & 0.836 & 100 & CAU & 83 & \\
\hline WP-09 & Saturado & 0.841 & 100 & CAU & 100 & \\
\hline WP-10 & Saturado & 0.782 & 100 & CAD & 100 & \multirow{5}{*}{$\begin{array}{l}\text { Resistenia } \\
\text { al cortante } \\
\mathrm{e}_{\text {aver. }}=0.78\end{array}$} \\
\hline WP-11 & Gaseoso & 0.797 & 100 & CAU & 95 & \\
\hline WP-12 & Gaseoso & 0.785 & 100 & CAU & 91 & \\
\hline WP-13 & Gaseoso & 0.798 & 100 & CAU & 82 & \\
\hline WP-14 & Saturado & 0.775 & 100 & CAU & 100 & \\
\hline WP-15 & Saturado & 0.715 & 100 & CAD & 100 & \multirow{6}{*}{$\begin{array}{l}\text { Resistencia } \\
\text { al cortante } \\
\mathrm{e}_{\text {aver. }}=0.71\end{array}$} \\
\hline WP-16 & Saturado & 0.703 & 100 & CAD & 100 & \\
\hline WP-17 & Gaseoso & 0.716 & 100 & CAU & 98 & \\
\hline WP-18 & Gaseoso & 0.706 & 100 & CAU & 91 & \\
\hline WP-19 & Gaseoso & 0.692 & 100 & CAU & 75 & \\
\hline WP-20 & Saturado & 0.706 & 100 & CAU & 100 & \\
\hline
\end{tabular}

CAU: consolidado anisotropicamente - No-drenado.

CAU: consolidado anisotrópicamente - Drenado.

Fuente: elaboración propia. 


\section{RESULTADOS}

\section{Línea de estado crítico}

Para la determinación de la línea de estado crítico, las muestras se consolidaron a esfuerzos más altos que los de estado crítico para una determinada relación de vacíos, asegurando que la respuesta del suelo fuera totalmente contractiva y de esta forma determinar la posición de la línea de estado crítico de manera confiable. La figura 3 presenta la localización de la línea de estado crítico y su posición con respecto a la relación de vacíos promedio de las muestras ensayadas.

Durante la ejecución de los ensayos, se midieron velocidades de onda de corte en varias etapas. En trabajos previamente realizados, Knai (2011) hizo mediciones con elementos bender en muestras alteradas preparadas con arena tomada del mismo sitio durante los años 2003 y 2004. En su trabajo, Knai encontró que los resultados obtenidos con elementos bender usando el método de pico-a-pico tuvieron una variación entre menos del 1,2\% de los resultados obtenidos a través de los métodos de correlación cruzada y de dominio de frecuencia. En lo que compete al trabajo presentado en esta investigación, los valores de velocidad de onda de corte que se reportan

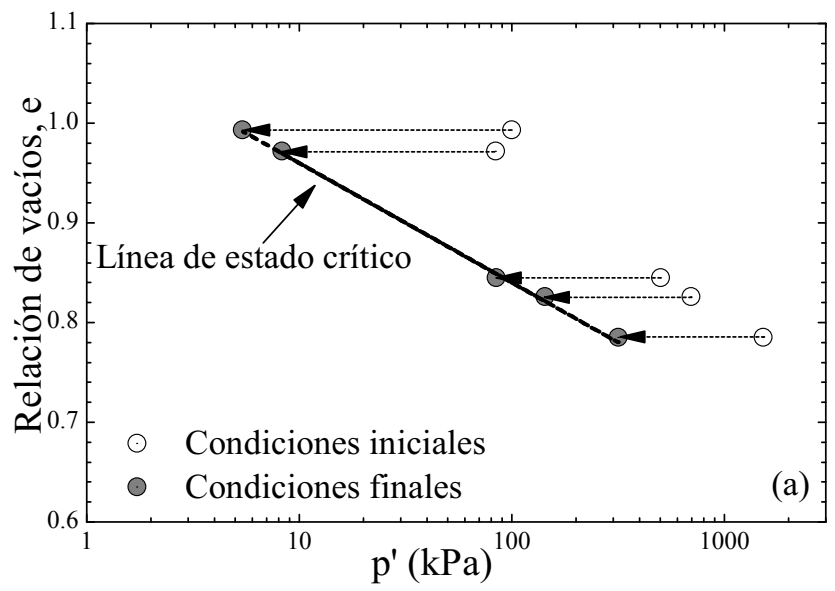

fueron determinados por medio de la metodología pico-a-pico.

La figura 4 presenta los valores calculados y normalizados del módulo elástico de corte $\left(\mathrm{G}_{\mathrm{BE}}\right)$ vs. el esfuerzo efectivo normal promedio. Estas mediciones fueron tomadas durante el proceso de consolidación usado para determinar la resistencia al cortante del suelo. En este programa de ensayos todas las muestra fueron consolidadas en condiciones saturadas. Como se esperaba, el $\mathrm{G}_{\mathrm{BE}}$ se incrementa a medida que se incrementa el esfuerzo efectivo normal promedio (Jung, Cho y Finno, 2007). El módulo elástico de corte se calculó de la siguiente manera:

$$
G_{B E}=\rho V_{B E}^{2}
$$

Donde, $\rho$ es la densidad total del suelo en el momento en que se midió la velocidad de onda de corte $\left(V_{B E}\right)$.

Para tener en cuenta la variación de la densidad del suelo durante el proceso de consolidación, los valores normalizados del módulo elástico de corte presentados en la figura 4 fueron correlacionados con las relaciones de vacíos de las muestras siguiendo el procedimiento presentado por Jung, Cho y Finno (2007). El resultado de esta correlación se expresa con la siguiente ecuación:

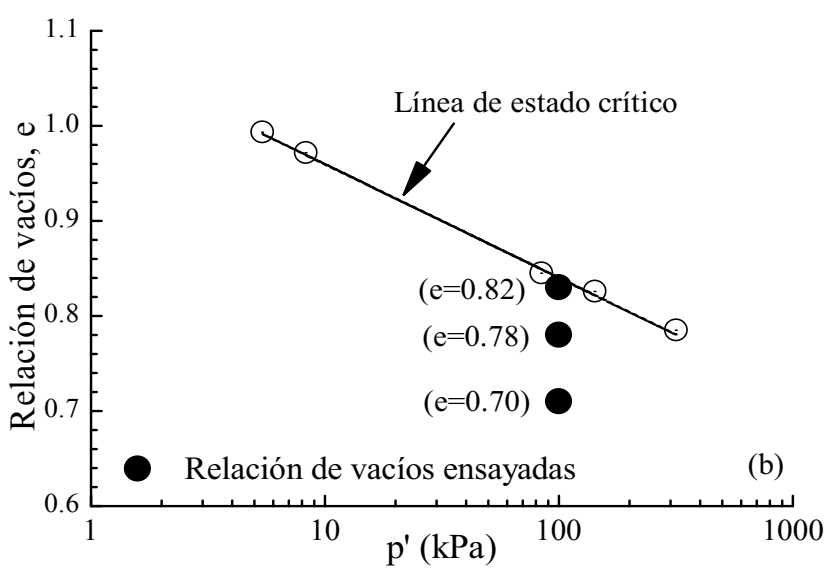

Figura 3. a) Línea de estado crítico (LEC) y b) posición de la relación de vacíos ensayada con respecto a la LEC.

Fuente: elaboración propia. 


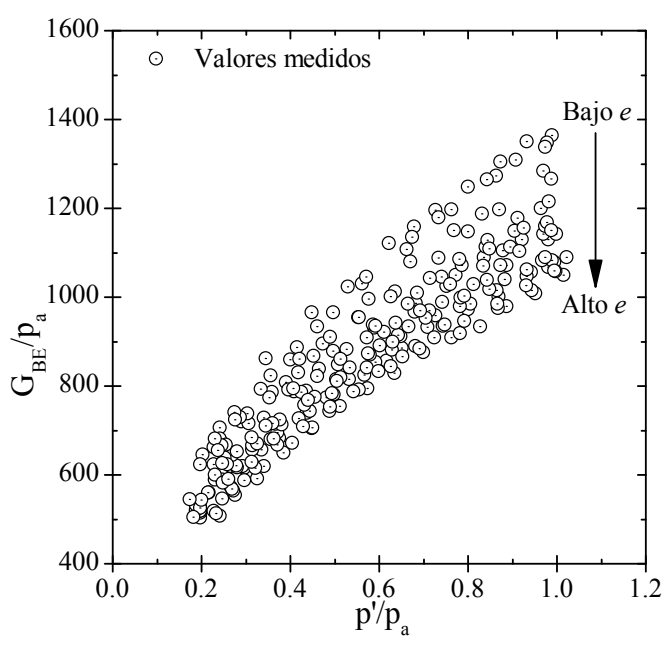

Figura 4. Resultados de las mediciones realizadas con elementos bender durante el proceso de consolidación de las muestras.

Fuente: elaboración propia.

$$
\frac{G_{B E}}{P_{a}}=A f(e)\left(\frac{p^{\prime}}{p_{a}}\right)^{i}
$$

Donde, $p_{a}=$ presión atmosférica $(101,3 \mathrm{kPa})$; $f(e)=$ función de relación de vacíos; y $A$ y $n=$ constantes del material. La figura 5a compara los valores calculados de $A f(e)$ basándose en las expresiones previamente publicadas de las mediciones de los valores de $A f(e)$ durante consolidación. Como se puede observar, los valores medidos de $A f(e)$ se encuentran dentro de las funciones de relación de vacíos propuestas por varios autores. La tabla 2 reúne las funciones de relación de vacíos, el promedio del valor $A$ y el coeficiente de determinación $\left(R^{2}\right)$ obtenido para siete (7) expresiones diferentes. Como se observa, el coeficiente de determinación
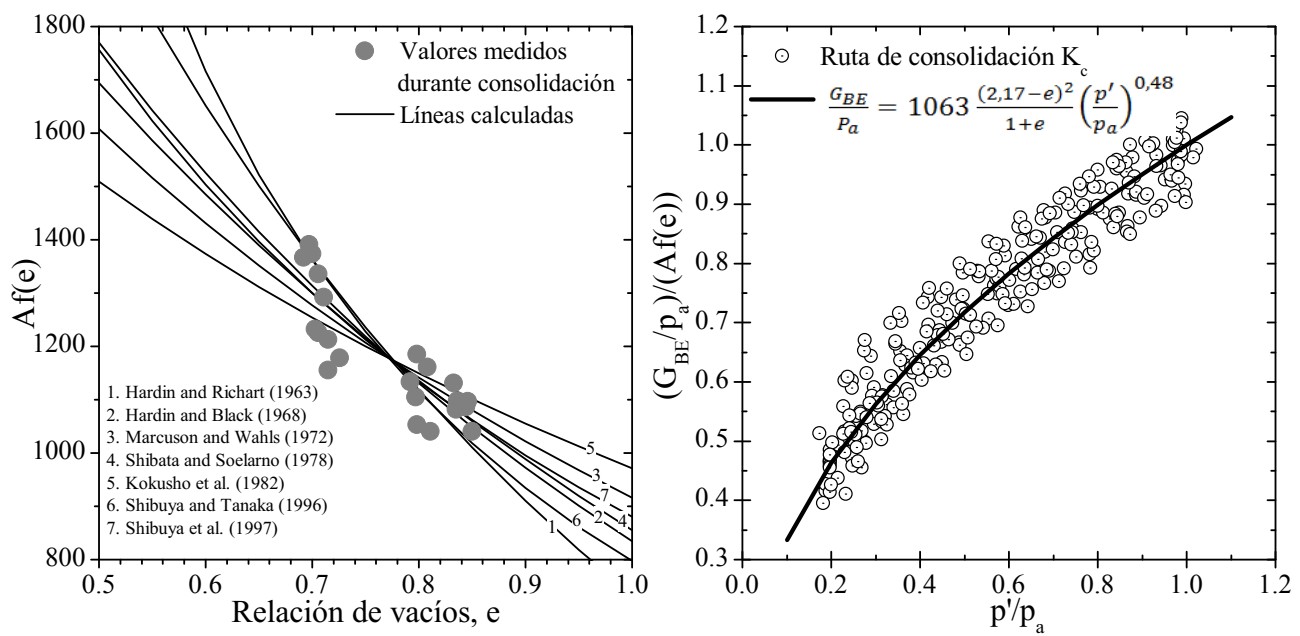

Figura 5. (a) Determinación de la función de relación de vacíos y (b) mejor función de ajuste de relación de vacíos Fuente: elaboración propia. 
varía muy poco entre las expresiones empíricas presentadas; por tanto, se escogió la función $f(e)$ propuesta por Hardin y Richart (1963) para los cálculos realizados en este trabajo, considerando principalmente que esta expresión fue desarrollada para suelos granulares. Igualmente, la figura $5 b$ compara los resultados medidos por medio de los elementos bender durante el proceso de consolidación con aquellos calculados por Hardin y Richart (1963). La expresión final obtenida para el módulo elástico de corte normalizado es:

$$
\frac{G_{B E}}{P_{a}}=1063 \frac{(2,17-e)^{2}}{1+e}\left(\frac{p^{\prime}}{p_{a}}\right)^{0,48}
$$

Tabla 2. Expresiones previamente publicadas de Af(e)

\begin{tabular}{|c|c|c|c|c|}
\hline$\#$ & Referencia & $f(e)$ & $\begin{array}{c}\text { Valor } \\
\text { promedio de A }\end{array}$ & $\begin{array}{l}\text { Coeficiente de } \\
\text { correlación. }\left(\mathbf{R}^{2}\right)\end{array}$ \\
\hline 1 & Hardin y Richart (1963) & $(2,17-\mathrm{e})^{2} /(1+\mathrm{e})$ & 1063,1 & 0,746 \\
\hline 21 & Hardin y Richart (1963) & $(2,91-\mathrm{e})^{2} /(1+\mathrm{e})$ & 454,4 & 0,745 \\
\hline 3 & Marcuson y Wahls (1972) & $(4,4-\mathrm{e})^{2} /(1+\mathrm{e})$ & 157,9 & 0,744 \\
\hline 44 & Shibata, T. y Soelarno (1978) & $0,67-e /(1+e)$ & 5005,5 & 0,743 \\
\hline 5 & Kokusho, Yoshida y Esashi (1982) & $(7,32-\mathrm{e})^{2} /(1+\mathrm{e})$ & 48,5 & 0,743 \\
\hline 6 & Shibuya y Tanaka (1996) & $\mathrm{e}^{-1,5}$ & 790,9 & 0,757 \\
\hline 7 & Shibuya, Hwang y Mitachi (1997) & $(1+e)^{-2,4}$ & 4621,5 & 0,748 \\
\hline
\end{tabular}

Las figuras 6 a 8 presentan las velocidades normalizadas de onda de corte durante el proceso de consolidación, repteo, desaturación y corte para las muestras ensayadas monotónicamente y con un promedio de relación de vacíos consolidada de $0,82,0,78$ y 0,71, respectivamente. Los valores de Vs calculados por medio de elementos bender se normalizaron con respecto al valor de $\mathrm{Vs}$ al final de la consolidación.

Los valores de Vs durante la etapa de desaturación fluctúan muy poco a medida que el dióxido de carbono disuelto en el fluido es forzado a salir de la solución. En general, la velocidad de onda de corte de las muestras de suelo gaseoso al final del proceso de desaturación, cuando las presiones del agua y del aire en la muestra son estables, varían en un rango de $\pm 5 \%$ con respecto a los valores después del repteo. Este pequeño cambio en $V s$ indica que la estructura inicial de la muestra no fue alterada significativamente durante el proceso de exsolución del gas. Estos resultados son consistentes con lo esperado, toda vez que los dos factores que influyen principalmente en la velocidad de onda de corte, relación de vacíos y esfuerzo promedio efectivo normal, no varían mucho durante el proceso de exsolución del gas.

La velocidad de onda de corte de las muestras ensayadas en condiciones no drenadas inicialmente disminuyó y posteriormente se incrementó mientras el proceso de corte continuaba. Este efecto se ve más pronunciado en las muestras más densas. En las muestras saturadas, la disminución inicial en Vs es el resultado de la generación de presiones de poros positivas al inicio del ensayo. El efecto del gas en la respuesta del suelo en condiciones drenadas y no-drenadas fue confirmado con las mediciones de velocidades de onda de corte. El gas hace que las velocidades de onda de corte obtenidas en condiciones no drenadas se acerquen a los valores de velocidades de onda de corte en condiciones drenadas. 


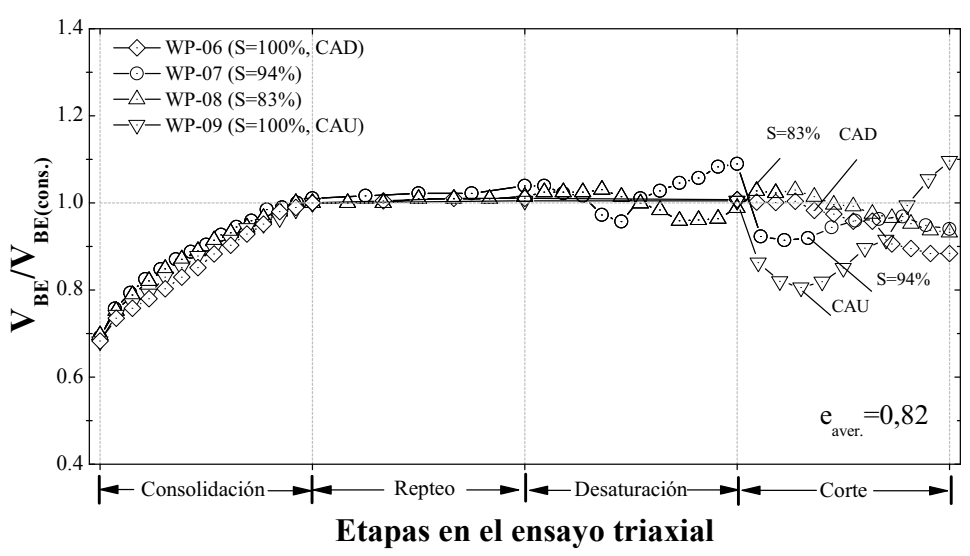

Figura 6. Velocidad de onda de corte normalizada durante el ensayo triaxial (Grupo 1, $\mathrm{e}_{\text {aver. }}=0,82$ )

Fuente: elaboración propia.

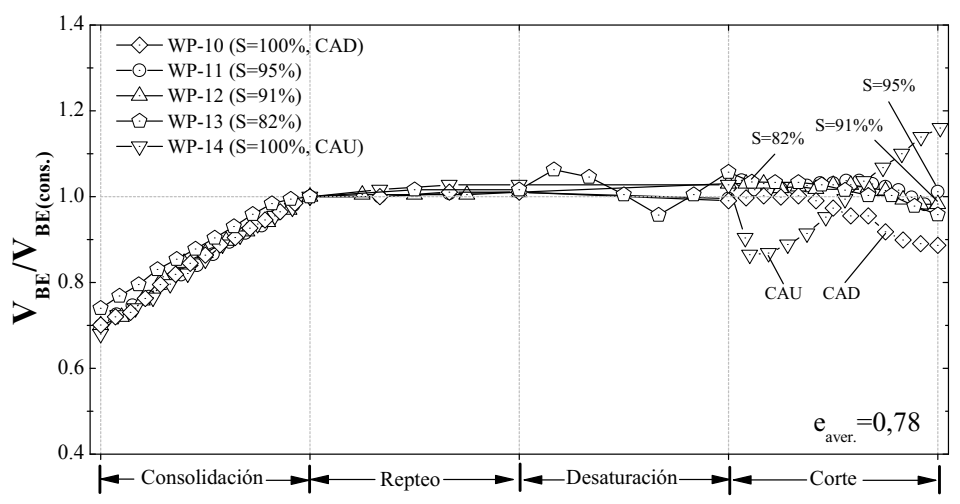

Etapas en el ensayo triaxial

Figura 7. Velocidad de onda de corte normalizada durante el ensayo triaxial (Grupo 2, $\mathrm{e}_{\text {aver. }}=0,78$ )

Fuente: elaboración propia.

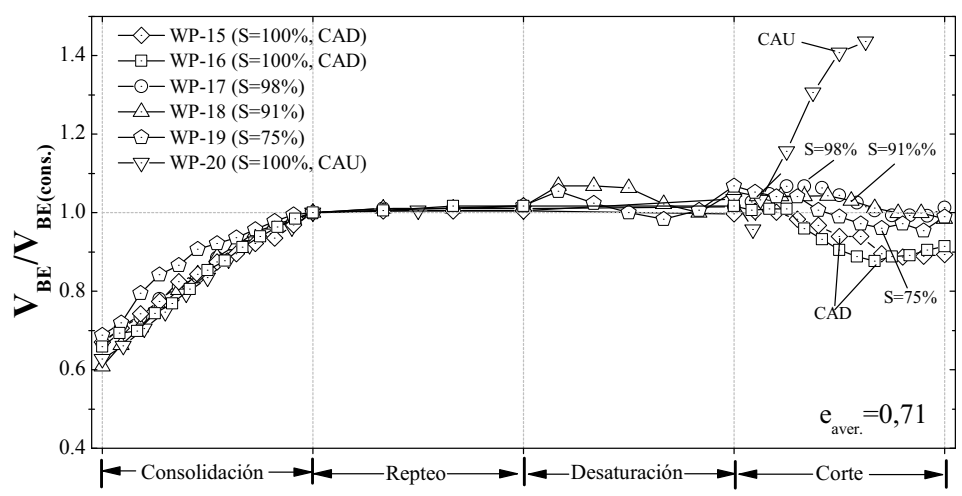

Etapas en el ensayo triaxial

Figura 8. Velocidad de onda de corte normalizada durante el ensayo triaxial (Grupo 3, $\mathrm{e}_{\text {aver. }}=0,71$ )

Fuente: elaboración propia. 


\section{CONCLUSIONES}

Los valores de velocidad de onda de corte obtenidos de los ensayos con elementos bender durante la etapa de consolidación están acorde a los resultados esperados; estos valores de velocidad se incrementan a medida que se incrementan los esfuerzos efectivos normales promedio, y difieren muy poco de las correlaciones de $A f(e)$ reportadas en la literatura técnica.

La velocidad de onda de corte medida durante el proceso de desaturación de la muestra, cuando se forza el dióxido de carbono disuelto en el fluido para que salga de la solución en forma de un gas ocluido, fluctúa muy poco durante esta etapa, indicando que las burbujas ocluidas desplazaron el fluido sin alterar la estructura interna de la muestra. Este resultado está acorde a lo esperado, ya que los dos factores principales afectando la velocidad de onda de corte son la relación de vacíos y el esfuerzo efectivo normal promedio. Adicionalmente, se puede concluir que el procedimiento propuesto es eficaz para remplazar el fluido por gas ocluido y de estar forma generar arenas gaseosas.

En general se puede observar que la presencia de gas, aún en muy pequeñas cantidades, afecta la respuesta de la velocidad de onda de corte cuando la muestra se ensaya bajo condiciones globales no drenadas (líneas cerradas). La velocidad de onda de corte de las muestras completamente saturadas inicialmente disminuye y luego incrementa a medida que el proceso de corte continúa, debido a que durante la primera fase del proceso de corte se desarrollan presiones de poro positivas y, por ende, se reducen los esfuerzos efectivos. El gas en forma ocluido tiene el efecto de cambiar la respuesta del suelo de condiciones no drenadas a condiciones drenadas, siendo más pronunciada la respuesta a medida que el suelo es más denso.

El gas tiene el efecto de inhibir el desarrollo de presiones de poros, tanto positivas como negativas, por tanto el incremento en la velocidad de onda de corte que se generan en las muestras densas debido al desarrollo de presiones de poros negativas se ve significativamente reducida por la presencia del gas; aún en pequeñas cantidades como es el caso de la muestra con $\mathrm{e}_{\text {aver. }}=0,71 \mathrm{y}$ $\mathrm{S}=98 \%$. Este comportamiento podría proveer una explicación del porque los valores de velocidad de onda de corte medidos en arenas densificadas con explosivos no incrementan significativamente con respecto a los valores medidos inicialmente.

\section{AGRADECIMIENTOS}

El trabajo experimental fue llevado a cabo en Northwestern University mientras el primer autor era un asistente de investigación en el Departamento de Ingenieria Civil y Ambiental. El primer autor agradece al profesor Richard Finno, profesor de Northwestern University, por sus valiosos comentarios y motivación durante el desarrollo de los ensayos. Ayuda financiera fue proveída por el Instituto de Infraestructura y Tecnología de Northwestern University y de la Fundación Nacional de Ciencias (National Science Foundation).

\section{REFERENCIAS BIBLIOGRÁFICAS}

Amaratunga, A. y Grozic, J.L.H. (2009). On the undrained unloading behaviour of gassy sands. Canadian Geotechnical Journal, 46, 1267-1276.

Been, K.; Jefferies, M.G. y Hachey, J. (1991). The critical state of sands. Géotechnique, 41(3), 365-381.

Castro, G.; Seed, R.B.; Keller, T.O. y Seed, H.B. (1992). Steady-state strength analysis of lower San Fernando Dam slide. Journal of Geotechnical Engineering, 118(3), 406-427.

Chaney, R. y Mulilis, J.P. (1978). Suggested method for soil specimen remolding by wet-raining. Geotechnical Testing Journal, 1(2), 107-108.

Chern, J.C. (1981). Effect of static shear on resistance to liquefaction. Tesis M.A.Sc. Vancouver, Canadá: The University of British Columbia.

Chern, J.C. (1985). Undrained response of saturated sands with emphasis on liquefaction and cyclic mobility. Tesis de doctorado. Vancouver, Canadá: The University of British Columbia. 
Finno, R.J.: Gallant, A.P. y Sabatini, P.J. (2016). Evaluating Ground Improvement after Blast Densification: Performance at the Oakridge Landfill. Journal of Geotechnical and Geoenvironmental Engineering, 142(1), 10.1061/(ASCE)GT.1943-5606.0001365, 04015054.

Ghionna, V. y Porcino, D. (2006). Liquefaction Resistance of Undisturbed and Reconstituted Samples of a Natural Coarse Sand from Undrained Cyclic Triaxial Tests. Journal of Geotechnical and Geoenvironmental Engineering, 132(2), 194-202. DOI:10.1061/(ASCE)1090-0241(2006)132:2(194)

Gohl, W.B.; Jefferies, M.G.; Howie, J.A. y Diggle, D. (2000). Explosive compaction: design, implementation and effectiveness. Géotechnique, 50(6), 657-665.

Grozic, J.L.H.; Imam, S.M.R.; Robertson, P.K. y Morgenstern, N.R. (2005). Constitutive modeling of gassy sand behaviour. Canadian Geotechnical Journal, 42(3), 812-829.

Hardin, B.O. y Black, W.L. (1968). Vibration modulus of normally consolidated clay. Journal of the Soil Mechanics and Foundations Division, 94(2), 353-370.

Hardin, B.O. y Richart, F.E.J. (1963). Elastic wave velocities in granular soils. Journal of the Soil Mechanics and Foundations Division, 89(1), 33-65.

Jung, Y.H., Cho, W. y Finno, R.J. (2007). Defining yield from bender element measurements in triaxial stress probe experiments. Journal of Geotechnical and Geoenvironmental Engineering, 133(7), 841-849.

Knai, H.B. (2011). Measuring the effect of occluded gas bubbles on stress-strain response of a loose to medium sand. Tesis de maestría. Evanston, IL: Northwestern University.

Kokusho, T.; Yoshida, Y. y Esashi, Y. (1982). Dynamic properties of soft clay for wide strain range. Soils Found., 22(4), 1-18.

Ladd, R.S. (1978). Preparing test speciments using undercompaction. Geotech Test J, GTJODJ., 1(1), 16-23.

Marcuson, W.F. y Wahls, H.E. (1972). Time effects on dynamicshear modulus of clays. Journal of the Soil Mechanics and Foundations Division, 98(12), 1359-1373.
Nageswaran, S. (1983). Effect of gas bubbles on the sea bed behaviour. Tesis de doctorado. Oxford University.

Narsilio, G.A. (2006). Spatial variability and terminal density: Implication in soil behavior. Tesis de doctorado. Atlanta, GA: Georgia Institute of Technology.

Narsilio, G.A.; Santamarina, J.C.; Hebeler, T. y Bachus, R. (2009). Blast Densification: Multi-Instrumented Case History. Journal of Geotechnical and Geoenvironmental Engineering, 135(6), 723-734.

Okamura, M.; Ishihara, M. y Tamura, K. (2006). Degree of saturation and liquefaction resistances of sand improved with sand compaction pile. Journal of Geotechnical and Geoenvironmental Engineering, 132(2), 258-264.

Okamura, M.; Takebayashi, M.; Nishida, K.; Fujii, N.; Jinguji, M.; Imasato, T.; ...; Nakagawa, E. (2011). InSitu Desaturation Test by Air Injection and Its Evaluation through Field Monitoring and Multiphase Flow Simulation. Journal of Geotechnical and Geoenvironmental Engineering, 137(7), 643-652. DOI: 10.1061/(asce)gt.1943-5606.0000483

Poulos, S.J.; Castro, G. y France, J.W. (1985). Liquefaction evaluation procedure. Journal of Geotechnical Engineering, 111(6), 772-792.

Ramos C., A.M. (2015). Influence of the void ratio and the confining on the static liquefaction in slopes in changi sand. Revista Tecnura, 19(43), 63-73.

Ramos C., A.M.; Felipe, P.-S.L. y Vega-Posada, C.A. (2016). Análisis de elementos finitos con un continuo elástico lineal tipo Cosserat. Revista Tecnura, 20(50), 43-54.

Shibata, T. y Soelarno, D.S. (1978). Stress-strain characteristics of clays under cyclic loading. Paper presented at the Proc., Japanese Society of Civil Engineering.

Shibuya, S.; Hwang, S.C. y Mitachi, T. (1997). Elastic shear modulus of soft clays from shear wave velocity measurement. Géotechnique, 47(3), 593-601.

Shibuya, S. y Tanaka, H. (1996). Estimate of elastic shear modulus in Holocene soil deposits. Journal of the Japanese Geotechnical Society : soils and foundation, 36(4), 45-55. 
Tomita, Y., Shima, A., \& Ohno, T. (1984). Collapse of multiple gas bubbles by a shock wave and induced impulsive pressure. Journal of Applied Physics, 56(1), 125-131.

Vaid, Y.P y Sivathayalan, S. (2000). Fundamental factors affecting liquefaction susceptibility of sands. Canadian Geotechnical Journal, 37(3), 592-606.

Vaid, Y.P.; Sivathayalan, S. y Stedman, D. (1999). Influence of specimen-reconstituting method on the undrained response of sand. Geotechnical Testing Journal, 22(3), 187-195.

Vega-Posada, C.A. (2012). Evaluation of liquefaction susceptibility of clean sands after blast densification. Tesis de doctorado. Evanston, IL: Northwestern Univ.

Vega-Posada, C.A.; Finno, R.J. y Zapata-Medina, D.G. (2014). Effect of Gas on the Mechanical Behavior of Medium-Dense Sands. Journal of
Geotechnical and Geoenvironmental Engineering, 140(11), http://dx.doi.org/10.1061/(ASCE)GT.19435606.0001163, 04014063. doi:10.1061/(ASCE) GT.1943-5606.0001163

Vega-Posada, C.A.; Zapata-Medina, D.G. y García-Aristázabal, E.F. (2014). Ground surface settlement of loose sands densified with explosives. Revista Facultad de Ingeniería, (70), 9-17.

Verdugo, R. e Ishihara, K. (1996). The steady state of sandy soils. Soils and Foundation, 36(2), 81-91.

Yegian, M.K.; Eseller-Bayat, E.; Alshawabkeh, A. y Ali, S. (2007). Induced-Partial Saturation for Liquefaction Mitigation: Experimental Investigation. Journal of Geotechnical and Geoenvironmental Engineering, 133(4), 372-380. doi: 10.1061/ (asce)1090-0241(2007)133:4(372) 